\title{
Coupling of polarisation of high frequency electric field and electronic heat conduction in laser created plasma
}

\author{
Eugene G. Gamaly and Andrei V. Rode \\ Laser Physics Centre, Research School of Physics \& Engineering, The Australian National University, Canberra, Australia \\ eugene.gamaly@anu.edu.au
}

\begin{abstract}
Powerful short laser pulse focused on a surface swiftly transforms the solid into the thermally and electrically inhomogeneous conductive plasma with the large temperature and dielectric permeability gradients across the focal spot. The laser-affected spot becomes thermally inhomogeneous with where temperature has maximum in the centre and gradually decreasing to the boundaries of the spot in accord to the spatial intensity distribution of the Gaussian pulse. Here we study the influence of laser polarization on ionization and absorption of laser radiation in the focal spot. In this paper we would like to discuss new effect in thermally inhomogeneous plasma under the action of imposed high frequency electric field. We demonstrate that high-frequency (HF) electric field is coupled with the temperature gradient generating the additional contribution to the conventional electronic heat flow. The additional heat flow strongly depends on the polarization of the external field. It appears that effect has maximum when the imposed electric field is collinear to the thermal gradient directed along the radius of a circular focal spot. Therefore, the linear polarised field converts the circular laser affected spot into an oval with the larger oval's axis parallel to the field direction. We compare the developed theory to the available experiments, discuss the results and future directions.
\end{abstract}

\section{Introduction}

The goal this paper is to examine the effects related to the direction of the vector of the electric field of the incident beam on the interaction process with inhomogeneous plasma. It is known that absorption of the laser energy and ionization of the dielectric depend on the polarisation state of the incident beam; however, these scalar entities naturally do not depend on the field direction. On the other hand, it is well known that the heat flow of electrons depends on the imposed external stationary electric/magnetic field. In the high frequency laser field the field direction changes sign during one period therefore it seems that HF field should not affect electrons diffusion process during the much longer time [1]. Indeed, as we see below, the field enters squared into the additional heat flow being non-zero after averaging over the many light periods.

\section{Heat flow in plasma placed in a high frequency polarised electric field}

Let's consider the interaction of polarized laser beam with plasma in equilibrium $\left(T_{e}=T_{i}=T\right)$. Monochromatic polarised laser beam, $\bar{E}=\overline{\boldsymbol{E}}_{\mathbf{0}} \cdot \exp \{-\boldsymbol{i} \boldsymbol{\omega} \boldsymbol{t}\}$, hits the vacuum-solid interface in the direction of normal to the surface. The unperturbed distribution function has a form of the conventional Boltzmann distribution: 


$$
f_{0}=A \cdot \exp \left\{-m v^{2} / 2 T\right\}
$$

Let's suggest that the increase of plasma temperature in the course of single pulse interaction is small in comparison to that in the initial state. Correspondingly, the perturbation of plasma distribution function is also small. The following kinetic equation describes thermally inhomogeneous electronic plasma placed in the high frequency field:

$$
\frac{\partial f}{\partial t}+\bar{v} \cdot \frac{\partial f}{\partial \bar{r}}-e \bar{E} \frac{\partial f}{\partial \bar{p}}=I_{\text {coll }}(\delta f)
$$

The perturbed distribution function reads:

$$
f=f_{o}+\delta f ; \delta f=\delta f_{t h}+\delta f_{\text {field }}
$$

The first term $\delta f_{\text {th }}$ in the perturbation relates to the thermal inhomogeneity (temperature gradient) while the second term $\delta f_{\text {field }}$ accounts for the field effect. In thermally homogeneous plasma the field perturbation has a form [1]:

$$
\delta f_{\text {field }}=\frac{e(\bar{E} \cdot \bar{v}) f_{0}}{T\left(i \omega-v_{e f f}\right)}
$$

The perturbation in Eq.(4) is responsible for the change in the dielectric permittivity of plasma at the action of the external field, which can be presented in the Drude-like form of the perturbed dielectric function. Keeping in mind the heat flow calculation, we express the total perturbation through the unperturbed distribution function as follows:

$$
\delta f=-\frac{\partial_{0}}{\partial \varepsilon} \xi=\frac{f_{0}}{k_{B} T} \xi
$$

We further take the total perturbation in the general form, $\boldsymbol{\xi}=\overline{\boldsymbol{g}} \cdot \nabla \boldsymbol{T}$. The vector function, $\boldsymbol{g}$, can be found from solution of Eq.(1), and in accord to the general rules it should include two true vectors, the field vector $\boldsymbol{E}$, and the velocity vector $\boldsymbol{v}$, in the same combination as in Eq.(4). The general expression for the true vector as a function of another two true vectors is the following:

$$
g_{\alpha}=g_{1} v_{\alpha}+E_{\alpha}(\bar{E} \cdot \bar{v}) g_{2}
$$

The electronic heat conduction coefficient expresses through this function, electron energy, $\varepsilon$, and velocity, $v_{a}$, in the form [1]:

$$
\kappa_{\alpha \beta}=-\frac{1}{k_{B} T} \int \varepsilon f_{0} v_{\alpha} g_{\beta} \cdot d^{3} p
$$

On the other hand the general expression of second order tensor constructed from the true vector, $\boldsymbol{E}$, is:

$$
\kappa_{\alpha \beta}=\kappa_{1} \delta_{\alpha \beta}-e_{\alpha} e_{\beta} \kappa_{2} ; \bar{e}=\frac{\bar{E}}{E}
$$

here $\boldsymbol{e}$ is the unit vector in the direction of the electric field; $\kappa_{l}$ and $\kappa_{2}$ are two scalar functions, which can be obtained from the solution of the kinetic equation. The heat flow in the presence of the field reads:

$$
q_{\alpha}=-\kappa_{\alpha \beta} \frac{\partial T}{\partial x_{\beta}}=\kappa_{1} \frac{\partial T}{\partial x_{\alpha}}-\kappa_{2} e_{\alpha} e_{\beta} \frac{\partial T}{\partial x_{\beta}}
$$


Note that field-related second term equals to zero when the polarisation direction is perpendicular to the temperature gradient. Let us now consider the effects of linear and circular polarisations in laserplasma interactions.

\section{Effect of the HF field on the heat conduction at different polarisations}

The intense laser pulse hitting the solid surface along the normal ( $z$ coordinate) produces hot plasma in the circular spot ( $\boldsymbol{x}, \boldsymbol{y}$ plane) of radius $\boldsymbol{r}_{f o c}$, surrounded by cold unperturbed target. The temperature has maximum in the centre of the circle while the temperature gradient is a maximum at the outer boundary of heated spot directed along the radius from the centre outside.

Linear polarisation. Let's consider the linear polarisation of the laser with the field direction along $\boldsymbol{x}$-axis. Then the field-affected heat flow part reads:

$$
q_{\text {field }}=\kappa_{2} e_{x}^{2} \frac{\partial T}{\partial x}
$$

Note that field affected flow exists in both directions only along the $\boldsymbol{x}$-axis, while in the other parts of heated circle heat flow is unaffected. Therefore, the expected effect of the linear polarisation is the elongation of the laser-affected spot in the direction polarisation, turning a circle into an oval with the large axis aligned along the direction of the electric field.

Circular polarisation. The electric field vector of the incident beam at any moment of time is collinear to the temperature gradient directed along the radius of the beam. Thus, the field influence on a heat flow is evenly distributed along the circle embracing the heated zone and leading to the change of the zone's radius.

\section{Quantitative estimates of the HF field effect through the scalar heat conduction coefficients}

In order to get a quantitative estimate of the effect one needs to know the value of scalar coefficient $\boldsymbol{\kappa}_{2}$. The scalar heat conduction coefficients have a conventional form:

$$
\kappa_{1} \approx \kappa_{2}=C_{e} n_{e} D_{e}
$$

where $\boldsymbol{C}_{\boldsymbol{e}}, \boldsymbol{n}_{\boldsymbol{e}}, \boldsymbol{D}_{\boldsymbol{e}}$ are respectively the electrons heat capacity $(\mathrm{J} / \mathrm{K})$, the electron number density and the diffusion coefficient $\left(\mathrm{cm}^{2} / \mathrm{s}\right)$. The diffusion coefficient expresses through the electron's velocity and momentum exchange frequency (as in Eq.(4)) in its general kinetic form:

$$
D_{e}\left[\mathrm{~cm}^{2} / s\right]=v_{e} \cdot l_{e} / 3=v_{e}^{2} / 3 v_{e f f} \propto v_{e}^{5}
$$

To evaluate the influence of the imposed electric field on the heat conduction one needs to know the changes in the electron velocity and the electron collision frequency. The velocity averaged by the distribution function, $\boldsymbol{v}_{e}^{\mathbf{2}}=\mathbf{2} \boldsymbol{k}_{\boldsymbol{B}} \boldsymbol{T}_{\boldsymbol{e}} / \boldsymbol{m}_{e}$, changes slightly during the short pulse. Thus it is likely that both scalar coefficients, one in the conventional heat conduction and another one in the HF field should be of the same order of magnitude. However, it is worthwhile to estimate changes in the velocity clarifying the physics of the effect. Rigorous but formal derivation of the HF field effect on the electronic heat transport in Section II has a clear physical interpretation. Indeed, the only force affecting the electrons' motion, which remains nonzero after averaging over many laser periods, is the gradient of the ponderomotive potential (or, the oscillation energy of a free electron), while the 
change in the initial plasma temperature by the imposed field is small. The equation of motion of the electron under this force reads:

$$
m_{e} \frac{d v}{d t}=-\frac{\partial}{\partial x} \frac{e^{2} E^{2}}{4 m_{e} \omega^{2}}
$$

where the oscillation energy, $\varepsilon_{o s}=\frac{e^{2} E^{2}}{4 m_{e} \omega^{2}}$, is averaged over the many periods for linear polarized field. Let's express the electric field through the intensity for linear polarisation, $\boldsymbol{E}^{\mathbf{2}}=\mathbf{8} \boldsymbol{\pi} \boldsymbol{I} / \boldsymbol{c}$. Then one gets for the oscillation energy, expressed through the laser wavelength in microns, $\varepsilon_{\text {osc }}=9.1 \cdot\left(I / 10^{14} \mathrm{~W} / \mathrm{cm}^{2}\right)(\lambda / \mu \mathrm{m})(\mathrm{eV})$. We take the spatial dependence of intensity across the focus in the Gauss form and suggest the scale of the ponderomotive force gradient is of the order of the focal spot radius. Then, from Eq.(9) one gets the estimate for the electron's velocity at the external boundary of the ablation spot:

$$
v_{e} \approx \frac{\varepsilon_{o s c} \cdot t_{p} \cdot 2 \cdot r \cdot \ln 2}{m_{e} r_{f}^{2}} .
$$

One can see that the electron velocity remains the same as during the action of a single pulse. Therefore the Coulomb collision frequency is also unchanged. However, the competition between the laser frequency (frequency of the electron's oscillation in the imposed field) and the collision frequency entered in Eq.(4) may affect $\boldsymbol{\kappa}_{2}$ coefficient. We neglect this difference as a first approximation taking, $\boldsymbol{\kappa}_{\boldsymbol{l}} \approx \boldsymbol{\kappa}_{2}$. Note, that in the field-affected heat conduction coefficient in Eq.(7), $\boldsymbol{e}_{\alpha} \times \boldsymbol{e}_{\beta} \times \boldsymbol{k}_{2}$, includes squared normalised field that should be averaged over many laser periods. The averaging linear polarized field (brackets) results in:

$$
<e_{\alpha} e_{\beta}>=\frac{1}{2}\left|e_{\alpha}\right| \cdot\left|e_{\beta}\right|
$$

where $\left|\boldsymbol{e}_{\alpha}\right|$ is the time envelope of the laser pulse. Thus, the field-affected diffusion coefficient should comprise a half of conventional diffusivity, $\boldsymbol{D}_{\boldsymbol{e}}^{\text {field }} \approx \boldsymbol{D}_{\boldsymbol{e}} / \mathbf{2}$. The additional heat wave moves along the field direction during the pulse on the distance:

$$
l_{\text {heat }}^{\text {field }} \approx\left(D_{e}^{\text {field }} t_{\text {pulse }}\right)^{1 / 2} .
$$

Let's suggest that the initial plasma state is a solid-density plasma at the temperature of several electron volts, this is the state created by the intense short laser pulse at the fluence above the ablation threshold. The diffusion coefficient in such plasma is around of $\boldsymbol{D}_{\boldsymbol{e}} \sim \mathrm{cm}^{2} / \mathrm{s}$. Thus, during a $100 \mathrm{fs}$ long pulse the heat wave affected by the field advances on the distance about a nanometer. One can see that the effect during the single short pulse action is small and it would be difficult to observe it experimentally. However, under the multiple-pulse action (at high repetition rate) the effects of many pulses might be accumulated, making the sum effect observable and measurable. The analysis of experiments presented below shows that there are indications of the influence of the state of polarisation on the heat conduction.

\section{Experiments: shape and size of laser-affected zone controlled by polarisation.}


It was demonstrated experimentally [2] that the size of the structure produced by high repetition rate laser scanned along the polymer surface depends on the relative directions of the electric field in the linear polarised laser beam and the scanning direction. Jonavicius et al. [2] have clearly shown that the width of the laser-produced line in a polymer equals to $667 \mathrm{~nm}$ when the electric field vector in linear polarised writing beam is perpendicular to the scanning direction (written line). When the field direction is parallel to the scanning direction and for the circularly polarised beam the line width was reduced to $597 \mathrm{~nm}$. The analysis performed in [3] shows that the combination of three phenomena might be responsible for the observed effect, namely, the accumulation of many consecutive pulses action, the tight focusing of the laser pulse and the field-affected heat conduction.

Experiments [2,3] were performed with $200 \mathrm{kHz}(280 \mathrm{fs}, 1030 \mathrm{~nm}, 0.986 \mathrm{~nJ}$ per pulse) laser beam scanned with velocity $10^{-2} \mathrm{~cm} / \mathrm{s}$. Therefore, around 1800 pulses hit the same area before the beam moves the distance equal to the laser spot on a writing surface. However, a single pulse delivers the fluence much less than the ablation threshold. The energy accumulation from more than 300 pulses occurs for overcoming the ablation threshold in the centre of the focal spot. Much more pulses are necessary for achieving the threshold at the edges of the focal spot with $0.9 \mu \mathrm{m}$ diameter. This phenomenon qualitatively explained [3] in accord with the energy accumulation effect experimentally observed and described in [4]. The HF field effect on the electronic heat conduction occurs in the plasma, which formed after several hundred pulses dwelling on the same focal spot. However during the short pulse time the outcome (the increase of the laser-affected spot in direction of the field) is around a nanometer. Therefore one may expect the HF heat conduction effect accumulation from many consecutive pulses in analogy with the energy accumulation. Our estimate of the the transformation of the original circular focal spot (red at Fig.1) into an oval with long axis of oval exceeding original focal diameter on several tens of nanometers is in qualitative agreement with observations [2].

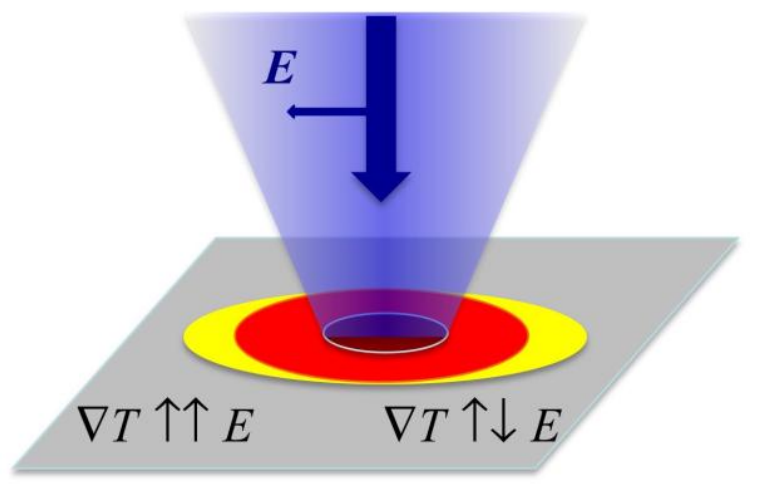

Fig.1. Qualitative illustration of the transformation of the s-polarised laser pulse illuminating circular focal spot (dark read) into a laser-affected oval on the surface (yellow) due to HF field effect on the heat conduction.

Another feature of the experiments in question [2] is the tight focusing of the laser beam with a microscope objective with high numerical aperture $N A=1.4$. It was found theoretically [5] but never observed experimentally that the energy density distribution in the focal spot of tightly focused (NA > 1) linearly polarized beam has elliptical form with the long axis in the field direction. The above effect might be enhanced by the increase in the electronic heat conduction (when polarization coincide with the temperature gradient) along with the increasing absorption. Overlapping of these 
effects results in the difference between two axes of the focal ellipse several tens of nanometers. However, it is difficult to separate the contributions of the tight focussing and the field affected heat conduction into the final line width.

\section{Conclusion}

Summing up, we demonstrate that the effect of HF electric field of the laser pulse on the electronic heat conduction in plasma has a clear physical justification and it is very likely that it was observed experimentally [2,3]. It is worth noting that these effects are of general nature and can be observed in any laser-matter interaction experiments where plasma produced by using tight focusing of linear-polarized light.

\section{Acknowledgements}

This research was supported under Australian Research Council's Discovery Project funding scheme (project DP120102980) and French Project for Scientific Cooperation (PICS 45052). Partial support to this work by Air Force Office of Scientific Research, USA (FA9550-12-1-0482) is gratefully acknowledged.

\section{References}

[1] Lifshitz, E.M., and L.P. Pitaevski, Physical Kinetics (Pergamon Press, Oxford, 1981).

[2] T. Jonavicius, M. Malinauskas and S. Juodkazis, Study on dependence of light polarization for direct laser writing in polymers (23rd ICO conference, Santiago de Compostela 26-29 August 2014).

[3] M. Malinauskas1, S. Rekstyte, T. Jonavicius, V. Mizeikis, E. Gamaly and S. Juodkazis, Coupling between polarization and thermal gradient in $3 D$ direct laser writing at tight focusing (submitted)

[4] B. Luther-Davies, A. V. Rode, N. R. Madsen, E. G. Gamaly, Picosecond high-repetition-rate pulsed laser ablation of dielectrics: the effect of energy accumulation between pulses, Optical Engineering 44(5), 051102 (May 2005)

[5] Ze'ev Bomzon, Min Gu, Joseph Shamir, Angular momentum and geometrical phases in tightfocused circularly polarized plane waves, Applied Pphysics Letters 89, 241104 (2006); see Fig.2. 